\title{
Beyond the No Free Lunch Principle: A New Combination of Arbitrage and Investment
}

\author{
Yi-Jang Yu \\ Department of Economics and Finance, Ming-Chuan University, Taiwan \\ Email: yjyu@mail.mcu.edu.tw
}

How to cite this paper: Yu, Y.-J. (2018) Beyond the No Free Lunch Principle: A New Combination of Arbitrage and Investment. Modern Economy, 9, 711-718. https://doi.org/10.4236/me.2018.94046

Received: March 14, 2018

Accepted: April 21, 2018

Published: April 24, 2018

Copyright $\odot 2018$ by author and Scientific Research Publishing Inc. This work is licensed under the Creative Commons Attribution International License (CC BY 4.0).

http://creativecommons.org/licenses/by/4.0/

\begin{abstract}
In the financial market, within both extremities of asset investment and arbitrage, there are sufficient rooms to contain something else other than speculation. The first aim of this study is thus to introduce a new sector which although cannot violate the common sense of "no free lunch" as can arbitrage, still is capable of surmounting general beliefs about the trade-off between risk and return that accompanies speculation. The second aim of this study is intended to reduce the ingredients of unfair games in the financial market.
\end{abstract}

\section{Keywords}

Asset Investment, Arbitrage, Speculation

\section{Introduction}

The two extremities in the finance market are asset investment and arbitrage. Currently, both are confronting with a difficulty of marginally decreasing return after all related know-how is close to maturity. What lies in between, speculation, can hardly attract followers who are deterred from its relatively high risk. The remaining question is: "Is there something else other than speculat ion?" The first aim of this study is thus to introduce a new game which, although cannot violate the common sense of "no free lunch" as can arbitrage, still is capable of surmounting the general belief about the tradeoff between risk and return accompanying speculation. As to the second main aim of this study it is an intention to reduce the ingredients of unfair games in the financial market.

For a better understanding, the second section of this paper will start with an illustration of basic individual stock investment. In the third section, a basic approach of option arbitrage will be demonstrated. Subsequently, a new game standing between the previous two will be demonstrated in the fourth section to 
reveal a surprising, potential new attraction in the financial market. Altogether, it should be sufficient for students in particular to obtain a basic understanding of primary money games in the financial market.

\section{A Simple Approach of Investment}

A fair gamble's winning chance must be exactly half. After applying more or less professional knowledge, investment should have a success chance of more than fifty percent but less than one hundred percent when there always have something that are not controllable.

Fundamental analysis is required to have a set of very diverse professional knowledge, including from microeconomics to macroeconomics, from supply to demand and so on. This is because factors affecting asset price movement are very diverse, and it is never an easy task to conduct a comprehensive study of them. If, instead, technical analysis would be applied, it is much simpler to practice when, usually, only transaction prices and, possibly, quantity data are analysed. Without any doubt, its efficacy and efficiency has long been questioned.

According to the concept of business cycle, there always have a number of different factors affecting one asset's price. These variables must be presented from different data sets which are measured in different time units, and their comprehensive analysis is hardly an easy job. This is why there are so many ideas of technical analysis suggested in the financial market and still no one can be the winner. It is thus advised by experienced investors that two or more tools of technical analysis should be applied simultaneously to deliver a better analytical outcome.

\subsection{A Simple WINDEX Approach with Upper and Lower Bounds}

If only one technical tool would be applied, then simplifying the analytical method is necessary. First, the analytical tool must be rational. The WINDEX will be applied in this study. It can be supported by certain economic theories including the principle of supply and demand [1], also can be flexibly extended into an EXCEL worksheet covering a sufficient range of WINDEX layouts. For a rational financial asset, the best applicable WINDEX can thus be evaluated and selected.

In reality, there is no guarantee that a positive return can be generated even if the success ratio of practice can be more than half. A fuzzy approach may be considered to possibly exclude most of unnecessary or erroneous execution with respect to small price movements. This can be easily achieved by employing an upper and a lower bound of WINDEX.

For example, in the Taiwan stock market, the price trend of " $2801 \mathrm{CHB}$ (Chang Hwa Bank)" from 2002/2/26 to 2004/1/14 in Figure 1 is less stable than its corresponding 30-day WINDEX trend in Figure 2 which, in turn, can be bounded within a range of $(0.3,0.7)$. Accordingly, by setting a lower bound of 0.4 and an upper bound of 0.6 , smaller fluctuation such as the one in circle " 5 " of 


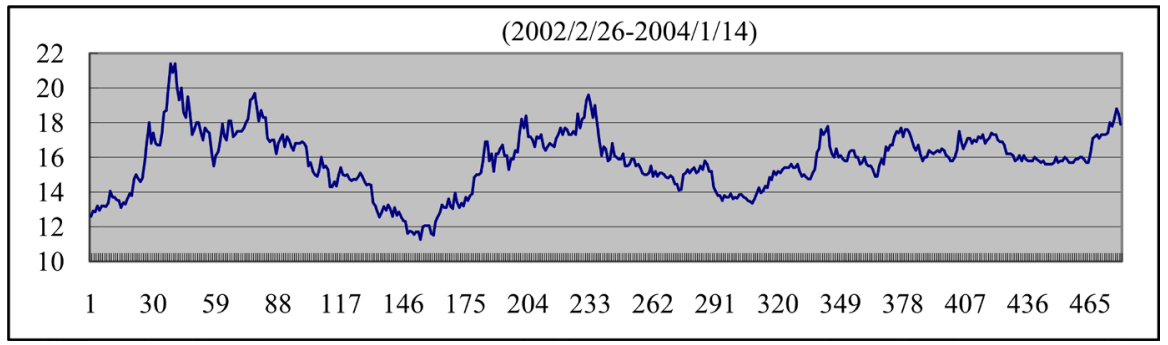

Figure 1. The stock price trend of the "CHB".

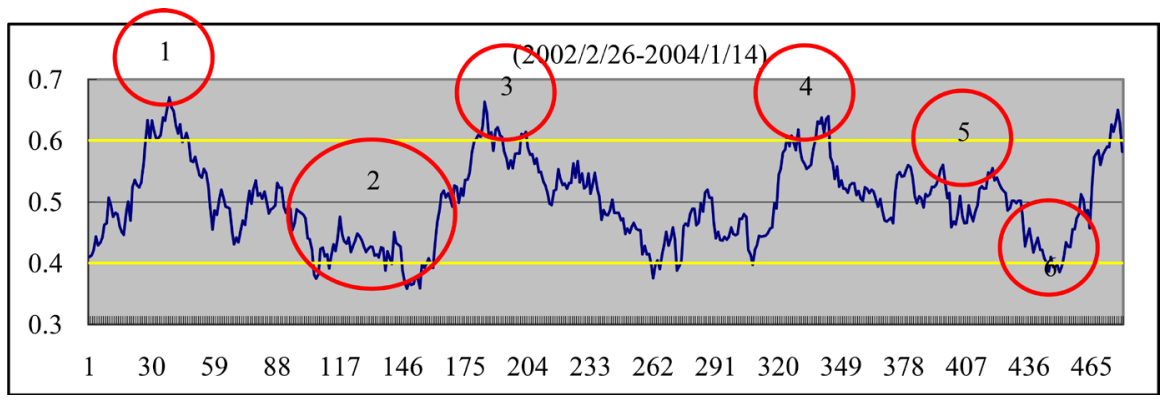

Figure 2. The 30-day WINDEX trend of the "CHB".

Figure 2 can be neglected. Moreover, in contrast to Figure 1, early response such as the one in circle " 6 " of Figure 2 can be detected.

For a rational stock, the applicable WINDEX can be trusted such that "what comes down (goes up), goes up (comes down)"; hence, the common approach of averaging the cost can be adopted. For example, in circle " 2 " of Figure 2, each time when the WINDEX falls below the lower bound " 0.4 " again, buy one more unit if the price would also be a new low. Similarly, whenever the upper bound of 0.6 is touched, short immediately after sell the stock, and short one more unit whenever the WINDEX rebounds over the upper bound.

\subsection{Simulation Results}

Daily "2801 CHB" stock data is obtained from the Taiwan Stock Exchange (TWSE) and is transformed into different columns of WINDEX as an EXCEL worksheet. In Table 1, column A is date; column B is the close price; cell C1 is the opportunity cost represented as the bond's current yield, about 0.0001 per day; other numbers in $\mathrm{C}$ column are daily rates of return in logarithmic form like "=LN(B3/B2)" in cell C3; column D is the 30-day WINDEX. As an example, the programming language in cell D32, representing the 30-day WINDEX on 2002/2/26, is arranged as: “=NORMSDIST ((AVERAGE (\$C3:\$C32) \$C\$1)/STDEV (\$C3:\$C32))"; columns E and F are the lower and upper bounds of 0.4 and 0.6 , respectively, and are chosen with limited experience.

After setting a lower and upper bounds, solving the problem of rebounding over either bound must be predetermined. For example, in circle " 2 ", “ 3 " and " 4 " of Figure 2, if the stock price can also be shown to have a new high or low each time of rebounding over the upper o lower bound, respectively, adding one 
Table 1. The EXCEL worksheet of WINDEX.

\begin{tabular}{ccccccc}
\hline & A & B & C & D & E & F \\
\hline $\mathbf{1}$ & 2801 CHB & Close & 0.0001 & 30-day WINDEX & Upper & Lower \\
$\mathbf{2}$ & $2002 / 1 / 4$ & 14.35 & & & & \\
$\mathbf{3}$ & $2002 / 1 / 7$ & 14.65 & 0.02069 & & & \\
$\mathbf{4}$ & $2002 / 1 / 8$ & 14.4 & -0.01721 & & & \\
$\mathbf{5}$ & $2002 / 1 / 9$ & 14.35 & -0.00348 & $:$ & $:$ & \\
$:$ & $:$ & $:$ & $:$ & $:$ & $:$ & $:$ \\
$:$ & $:$ & $:$ & $:$ & & & \\
$\mathbf{3 0}$ & $2002 / 2 / 22$ & 13.1 & -0.01515 & & & \\
$\mathbf{3 1}$ & $2002 / 2 / 25$ & 12.6 & -0.03892 & 0.4099 & 0.4 & 0.6 \\
$\mathbf{3 2}$ & $2002 / 2 / 26$ & 12.6 & 0 & 0.4123 & 0.4 & 0.6 \\
$\mathbf{3 3}$ & $2002 / 2 / 27$ & 12.9 & 0.02353 & 0.4205 & 0.4 & 0.6 \\
$\mathbf{3 4}$ & $2002 / 3 / 1$ & 12.85 & -0.00388 & 0.4432 & 0.4 & 0.6 \\
\hline 35 & $2002 / 3 / 4$ & 13.2 & 0.026873 & & & \\
\hline
\end{tabular}

Data source: The Taiwan Stock Exchange Corporation (TWEX); author.

more position to even out the cost will be automatically executed.

For convenience, no transaction cost is considered. Unless the performance is poor, otherwise, impact from transaction costs would not be significant when it is already quite low in the Taiwan stock market. Furthermore, although stock dividends in 2002 were 0.4 for " 2801 CHB", it is none in 2003. Therefore, again for convenience, no stock dividend would be adjusted in the simulation test. Finally, it is assumed that all required transactions can be exactly executed with the close price.

Main events and important information during the testing period from 2002/2/26 to 2004/1/14 were listed in the Appendix. On the first date of $2002 / 2 / 26$, the 30-day WINDEX was just broken through the lower bound from below to become 0.4099 , a buy signal would be taken. On 2002/4/9 and 4/10 the WINDEX went down first and rebounded to break through the second time the upper bound 0.6, the stock price was also a new high, short one more unit automatically. Later on, the same scene occurred again on 2002/4/30 and 5/2, the same plot was executed once more. Subsequently, when the lower bound 0.4 was touched on $2002 / 7 / 25$, those three existing short positions were liquidated and one new unit was bought immediately.

Finally, since the whole simulation test was close to its end on 2004/1/14 when the previous long position was liquidated, no more action would be followed. The performance was a gross of 32.1. In contrast, the simple buy-and-hold strategy could only provide 5.4. In order to possibly avoid the interruption of market close during the long holidays of Chinese New Year, an identical testing period from $2 / 15$ of the first year to $1 / 14$ of the third year was chosen for the next ten years. Based on the same instructions, the simulation performance 
could be shown to have: 2004-06, 6.95; 2006-08, 16.35; 2008-10, 9.55; 2010-12, $11.35 ; 2012-14,9.6$. Comparing to the simple hold-and-sell strategy whose outcome could only be: 2004-06, $-1.5 ; 2006-08,2.05 ; 2008-10,-3.1 ; 2010-12,1.65$; 2012-14, 2.15, the deviation is significant. This demonstrates that, for a rational or matured stock, the simple WINDEX approach with lower and upper bounds can possibly achieve a much better performance.

\section{A Simple Approach of Arbitrage}

The common sense of "no free lunch" is irrelevant when arbitrage can reach a success possibility of one hundred or near one hundred percent. For example, for two assets A and B having an identical flow of future return, their market prices must be identical or very close to each other. If asset A's price is significantly higher than B's, then one should short A plus buy B and still can deposit some remains. Since asset A's future return can be exactly covered by asset B, the remained plus possible interest accrued are arbitrage profits.

Quasi-arbitrage on the space dimension incurs certain costs, can be deemed as the predecessor of trade; on the time dimension, an example was food purchased with low prices during bumper crop years and sale them with high prices during famine years by Egyptian Pharaohs. At present, arbitrage with space and time dimensions can be realised via financial derivatives.

Based on the put-call parity relationship, both call and put having identical exercise terms can be arranged to produce a synthesized future contract. However, the actual future contract has its own supply and demand. Therefore, both actual and synthesized future would most likely move inconsistently especially on the opening second of all related option and future market and hence provide an opportunity of arbitrage. Though this kind of opportunity would be exploited and mostly seized in seconds, the appearance of this kind of arbitrage opportunity cannot be avoided when all related option and future markets are not running continuously. The good news is that all related professional knowledge has long been disseminated, and the exploitation of this kind of arbitrage opportunity already faces fierce competition.

The analysis object in this study is the Taiwan Stock Index Options (TXO), its underlying asset is the Taiwan Stock Index Futures (TX). Taking two sets of TXO call and put on 2008/1/2 as an example, each has an exercise price of 8300 or 8500 , respectively, but with an identical expiration month in February. All open and close prices are listed in Table 2. By combining 8300 long call with 8300 short put, a long future can be synthesized with a contract price of $(8300+410-290)=8420$. Similarly, by combining 8500 short call with 8500 long put, a short future can be synthesized with a contract price of $(8500+345$ $-340)=8505$. In the end, a gross of arbitrage, $8505-8420=85$, can be expected if holding all positions till the expiration date. However, liquidating all positions on the same day with close prices can reveal a gross arbitrage profit of $85+2=87$. 
Table 2. Market data of TXO.

\begin{tabular}{ccccccccc}
\hline Date & Contract & Expiration month & Exercise price & Position & Open & High & Low & Close \\
\hline $2008 / 1 / 2$ & TXO & 200,802 & 8300 & call & 410 & 410 & 337 & 346 \\
$2008 / 1 / 2$ & TXO & 200,802 & 8300 & put & 290 & 350 & 265 & 350 \\
$\ldots$ & $\ldots$ & $\ldots$ & $\ldots$ & $\ldots$ & $\ldots$ & $\ldots$ & $\ldots$ & $\ldots$ \\
$2008 / 1 / 2$ & TXO & 200,802 & 8500 & call & 345 & 345 & 240 & 240 \\
$2008 / 1 / 2$ & TXO & 200,802 & 8500 & put & 340 & 446 & 340 & 446 \\
\hline
\end{tabular}

Data source: The Taiwan Future Exchange (Taifex).

Table 3. Daily market data of TX.

\begin{tabular}{ccccccc}
\hline A & B & C & D & E & F & G \\
\hline Date & Open & High & Low & Close & Open $_{\mathrm{t}}-$ Close $_{\mathrm{t}-1}$ & Close $_{\mathrm{t}}-$ Open $_{\mathrm{t}}$ \\
\hline $2015 / 7 / 14$ & 9040 & 9084 & 8982 & 8984 & & -56 \\
$2015 / 7 / 15$ & 9040 & 9078 & 8989 & 9030 & 56 & -10 \\
$2015 / 7 / 16$ & 8935 & 8983 & 8900 & 8918 & -95 & -17 \\
\hline
\end{tabular}

Data source: The Taiwan Future Exchange (Taifex).

\section{A Simple Approach of the New Game}

Every monetary game in a closed environment must have an outcome of zero-sum, and risk-reduction from one player would automatically become the loss of the other. Once the asymmetry of assuming risks by either player exists, and its impact can be further augmented by the leverage effect inherited with the options and futures market, unfair games would be created accordingly.

The analysis object here is still the TXO. In Table 3, all related data are listed in the EXCEL form, and columns F and G are what the evaluation bases for establishing positions. The way of evaluation is straight forward. For example, on $2015 / 7 / 14$ the G column has a value of " -56 " (=8984 - 9040), but on the next trading date $7 / 15$ the F column has a positive value of " 56 " (=9040 - 8984), showing no consistent momentum hence no action is taken. A similar negative value is also revealed in the G column on $7 / 15$, but this time it is followed by another negative value in the $\mathrm{F}$ column on $7 / 16$. Consequentially, the nearest out-of-the-money put will be established. Similarly, if both G column on today and $\mathrm{F}$ column on tomorrow can be shown to have positive values, the nearest out-of-the-money call should be chosen after liquidating the previous put position.

Based on the assumption that all transactions can be exactly executed with "open" prices during the whole testing year of 2015, simulation results can be shown to have 23 times of success out of a total number of 54 trials; a poor ratio of a little more than $40 \%$. However, after deducting the total cost of establishing all positions, 7587, from the total revenues of liquidating all positions, 8757 , the gross profit is a surprising 1170 . The highest cost of establishing a position is 350 occurred on $9 / 2$, meaning that the gross rate of return could possibly be very 
high. Since total transaction fees and taxes are relatively small, they could not significantly affect the performance.

One reminder is that only positions with a maturity date in the next month will be established whenever moving into a new month. This is a consideration that can avoid a possible fault of existing position. Moreover, since lowering risk can always raise the performance in the new game, it is thus suggested that those dates having too high premiums could be abandoned. After excluding those three dates having highest premiums: 9/2,350;9/3, 273; 9/7,315, an additional return of 396 can be saved.

\section{Unfair Games}

It is well known that long options assume limited losses but not short options, and this kind of risk asymmetry cannot be effectively adjusted by current approach of option pricing. For option pricing, an extremely large loss is only deemed as a rare possibility in a fixed future time. However, in actual deal, an extremely large loss could possibly occur earlier and immediately terminate the deal. To neglect this kind of time asymmetry would underestimate the risk assumed by the short parties in the option market. Once this kind of unjustified treatment can be further augmented by the leverage effect, unfair games can be easily realized in the market.

For all arbitrage opportunities that can be created in the options market, it is apparently a violation of the principle of no-arbitrage pricing. But in essence, it is a concern of the efficacy of the options market. By combining spot and related future contracts, possible opportunities of arbitrage can already be locked in. When options could be maneuvered to produce tremendously more arbitrage opportunities and becomes the objects of zero-sum games, and when opportunities costs to be generated accordingly, including costs of misallocation of resources and waste of human wisdom, can far surpass its initial benefits of risk-hedging, the rationality of having options ought to be reevaluated.

\section{Conclusions}

In the financial market professional knowledge has its own supremacy. However, related knowledge has long been matured in the arbitrage and investment worlds; new territories must be exploited. The new game introduced in this study can be a new attraction when it is capable of violating a general belief about the tradeoff between risk and return. However, even if professional knowledge could be deemed as a part of national wealth, if it could not contribute to the growth of real economy or the enhancement of social welfare, it would probably be declined to become a tool of monetary games. Exertion of human wisdom must balance private and public interests; otherwise, it could be mostly wasted.

In the world of option arbitrage, opponents of arbitrageurs are disadvantageous. In the new game introduced in this study, short positions are disadvanta- 
geous. The former has a problem of perfect substitution between different sets of option combination, even including related futures; the latter has structural problem in the job of option pricing. Before these defects could be improved, permitting unfair games to exist in the financial markets must be irrational. Resistance from vested interests could be obvious; therefore, to accelerate the dissemination speed of related knowledge may be a good idea to reduce the ingredient of unfair games in the financial market and to avoid the waste of human wisdom.

\section{References}

[1] Yu, Y. (2015) Short-Term Technical Predictive Ability in the Taipei Stock Market. Modern Economy, 6, 12-20. https://doi.org/10.4236/me.2015.61002 